\title{
Conhecimento de Discentes do Curso de Graduação em Medicina sobre Vias de Parto
}

\section{The Undergraduate Medical Students' Knowledge about Ways of Childbirth}

\author{
Yasmin Sendrete de Carvalho Oliveira Leite ${ }^{I}(\mathbb{D})$ \\ Erika Cristina Napolitano Giuliano ${ }^{I} \mathbb{D}$ \\ Sérgio Alves Dias Júnior ${ }^{I}(\mathbb{D}$ \\ Marcela Souza da Silva ${ }^{I}$ D \\ Fábio de Souza Terra ${ }^{I}(\mathbb{D}$ \\ Patrícia Mônica Ribeiro ${ }^{I}(\mathbb{D}$
}

\section{PALAVRAS-CHAVE}

- Cesariana.

- Parto Normal.

- Saúde da Mulher.

- Estudantes de Medicina.

\section{RESUMO}

Introdução: As taxas de cesáreas no mundo todo têm apresentado valores acima do recomendado pela Organização Mundial da Saúde, especialmente no Brasil, e, com isso, trata-se de um problema de saúde pública. As principais causas para esse cenário se encontram na orientação e no suporte fornecidos pelo profissional de saúde, principalmente pelo médico. Assim, a formação do discente de Medicina pode impactar o seu perfil como profissional. Este estudo teve como objetivo avaliar o perfil e o conhecimento de acadêmicos do curso de Medicina de uma universidade pública acerca das vias de parto. Método: Trata-se de um estudo quantitativo de natureza descritiva e de corte transversal que se desenvolveu em uma universidade pública do sul de Minas Gerais com 165 acadêmicos do curso de Medicina, do quarto, sexto, oitavo e décimo períodos. Para a coleta de dados, aplicou-se um questionário semiestruturado, contendo 57 questões, após a aprovação do Comitê de Ética em Pesquisa. Os dados foram tabulados e avaliados por meio de frequência e também expressos em porcentagem. Resultados: Constatou-se que 89,09\% dos estudantes acertaram a indicação de via de parto no caso de gestantes de baixo risco sem intercorrências, já para a via de parto de gestantes diabéticas e com pré-eclâmpsia, apenas $25,45 \%$ e $18,18 \%$, respectivamente, acertaram. Verificou-se também que $75,15 \%$ dos estudantes consideram que o uso de mais tecnologia durante o parto o torna mais benéfico para a mãe e o bebê, e 77,58\% dos participantes não indicariam a cesariana por causa das comodidades. Conclusões: Podese concluir que existem discrepâncias quanto às respostas obtidas pelos participantes do estudo. É importante que novas pesquisas sejam feitas para avaliar as fragilidades na matriz curricular visando formar profissionais médicos que possam contribuir para a mudança do cenário obstétrico do país. 


\section{KEYWORDS}

- Cesarean Section

- Natural Childbirth.

- Women's Health.

- Students Medical.

Recebido em 27/6/20

Aceito em 29/9/20

\section{INTRODUÇÃO}

A cesárea é um procedimento que pode salvar a vida de mães e bebês se for realizada quando há indicação, pois, trata-se de um procedimento cirúrgico invasivo com diversos riscos imediatos e de longo prazo. A Organização Mundial da Saúde (OMS) concluiu que uma taxa de cesáreas maior do que $10 \%$ não apresenta melhora nos índices de mortalidade materna e neonatal, não justificando o aumento da realização de tal intervenção. Portanto, passou a recomendar que os esforços visassem garantir que a cesariana seja realizada apenas quando estritamente necessária em vez de buscar uma taxa específica para ela ${ }^{1}$.

Para o Brasil, a taxa de cesárea recomendada pela OMS é de $25 \%$ a $30 \%{ }^{2}$. Porém, as taxas nacionais são superiores a esses valores, chegando a mais de $50 \%$ de todos os nascimentos no país. Nos setores privados de atenção à saúde, por exemplo, as cesáreas representam $84,6 \%$ dos partos realizados ${ }^{3}$. Manter tal padrão de nascimento é um problema de saúde pública, pois, além de o procedimento ser mais caro, há a exposição de muitas mulheres a riscos de intercorrências que oneram ainda mais o Estado ${ }^{4}$. Além dos custos, a operação cesariana possui maior morbimortalidade para a gestante quando comparada ao parto vaginal, uma vez que apresenta um risco maior para ocorrência de infecção, rotura uterina e morbidade febril puerperal. Com relação aos efeitos da cesárea nos recém-nascidos, há discussões sobre complicações geradas durante a cesárea eletiva, como taquipneia transitória do recém-nascido e desconforto respiratório neonatal ${ }^{5}$.

De forma geral, quando se analisam as principais causas da epidemia de cesáreas, pode-se observar que se trata de um problema multifatorial. Dentro dessas causas, encontramos o papel da orientação e do suporte fornecido pelo profissional de saúde, especificamente o profissional médico, durante a escolha do tipo de parto a ser realizado, especialmente quando essa orientação não segue as indicações para a realização de cesáreas ${ }^{4}$.

As Diretrizes Curriculares do Ministério da Educação de 2001 que norteavam o curso de Medicina visavam à formação de um profissional

\section{ABSTRACT}

Introduction: The cesarean section rates worldwide, and especially in Brazil, have exceeded the levels recommended by the World Health Organization, therefore constituting a public health problem. The main causes of this scenario lie in the guidance and support provided by the health professional, especially the doctor. Thus, the training of medical students can impact their profile as a professional. The objective of this study was to evaluate the profile and knowledge of medical students at a public university regarding the different methods of childbirth. Method: This is a quantitative, descriptive and cross-sectional study that was developed at a public university in the south of Minas Gerais, involving 165 second, third, fourth and fifth year medical students. For the data collection a semi-structured questionnaire containing 57 questions was applied, following approval by the Research Ethics Committee. The data were tabulated and evaluated by frequency and expressed as numerical percentages. Results: It was found that $89.09 \%$ of the students correctly indicated the mode of delivery in the of low-risk pregnant women without complications, whereas only $25.45 \%$ and $18.18 \%$ got the mode of $75.15 \%$ of the students considered that the use of more technology during childbirth is beneficial for both mother and baby, and $77.58 \%$ of would not recommend a cesarean section for reasons of convenience. Conclusions: It can be concluded that there are discrepancies in relation to the answers obtained in the study. It is important that further research is carried out to assess weaknesses in the curriculum with a view to training medical professionals in line with the changing obstetric scenario in Brazil. 
Assim, este estudo teve como objetivo avaliar o perfil e o conhecimento de acadêmicos do curso de Medicina de uma universidade pública acerca das vias de parto.

\section{MÉTODO}

\section{Tipo de estudo}

Trata-se de um estudo com abordagem quantitativa de natureza descritiva e de corte transversal no que concerne à temporalidade.

\section{Participantes e local de estudo}

Participaram do estudo 165 discentes de Medicina de uma universidade pública do sul de Minas Gerais: 46 do quarto período, 50 do sexto, 48 do oitavo e 21 do décimo. De um total de 200 alunos matriculados nos períodos descritos que foram convidados a participar do estudo, 165 se voluntariam para tal.

Trata-se de amostra por conveniência cujos critérios de inclusão foram: ser estudante de qualquer idade e sexo e estar devidamente matriculado nos períodos-alvo do estudo. Excluíram-se os discentes que estavam afastados das atividades acadêmicas por algum motivo de saúde ou por trancamento de matrícula.

Procedimento e instrumento de coleta de dados

Os dados foram coletados por meio da aplicação de um questionário, elaborado pelos pesquisadores, composto por 57 perguntas fechadas. Os participantes deste estudo foram orientados a preenchê-lo indicando com um $\mathrm{X}$ as afirmações consideradas corretas e que julgavam de maior coerência com a sua vivência e seus conhecimentos. No questionário, havia duas partes: caracterização dos participantes do estudo e perguntas referentes à via de parto.

$\mathrm{O}$ referido instrumento foi elaborado com base na literatura e apresentou conceitos estabelecidos pelo Ministério da Saúde e pela OMS. Dois juízes da área foram os responsáveis pela avaliação do questionário e pelas sugestões de mudanças, procedimento realizado para garantir melhor refinamento e entendimento do instrumento.

Realizada em sala de aula durante horário acadêmico e em espaço cedido pelo docente responsável da disciplina, a coleta ocorreu no segundo semestre de 2018 e no primeiro semestre de 2019. Os discentes inicialmente receberam explicações sobre a pesquisa. Em seguida, entregaram-se aos que concordaram em participar o questionário e o Termo de Consentimento Livre e Esclarecido (TCLE) em duas vias. Por fim, o questionário devidamente preenchido e uma das vias do TCLE foram recolhidos pelos pesquisadores e arquivados para análise posterior.

\section{Tratamento dos dados}

Para a análise dos dados gerados, elaboraram-se tabelas, com auxílio do aplicativo Microsoft Excel 2010, para cada item/pergunta do questionário. Os dados obtidos foram tabulados e avaliados por meio de frequências e expressos em porcentagem para discussão e verificação dos objetivos propostos.

\section{Aspectos éticos}

O estudo foi realizado após a devida aprovação do Comitê de Ética em Pesquisa da Universidade Federal de Alfenas (Unifal), de Minas Gerais, tendo sido aprovado com Parecer n ${ }^{\circ} 2.594 .862$, e também após o consentimento e a autorização da referida universidade para a realização da pesquisa. Os acadêmicos somente fizeram parte da pesquisa quando concordaram em participar, após as explicações sobre a origem e objetivo deste estudo e a assinatura do TCLE, em conformidade com a Resolução no 466/2012 do Conselho Nacional de Saúde.

\section{RESULTADOS}

De acordo com os resultados obtidos, dos 165 discentes participantes, a maioria estavam na faixa etária de 18 a 22 anos $(48,48 \%)$, seguida da faixa de 23 a 27 anos (42,42\%). Quanto ao sexo, predominou o feminino (57,58\%), e o masculino representou $42,42 \%$. Com relação ao estado civil, 93,94\% eram solteiros; e 5,45\%, casados. Dentre os participantes, $97,58 \%$ não tinham filhos, e apenas $2,42 \%$ já eram pais.

Quanto às disciplinas com temas voltados às humanidades, a maioria dos discentes confirmou a presença delas nas matrizes curriculares (98,79\%). Com relação ao número de disciplinas de humanidades já cursadas, 55,76\% afirmaram que já haviam participado de cinco ou mais, e $83,79 \%$ relataram que haviam gostado delas. Para $67,88 \%$ dos estudantes avaliados, as disciplinas de humanidades exigiam menos quando comparadas às disciplinas técnicas (Tabela 1 ).

Um grupo de questões tinha como enfoque o conhecimento dos participantes acerca das indicações sobre as vias de parto, bem como sobre outras temáticas pertinentes. As questões com maiores índices de

\begin{tabular}{|c|c|c|}
\hline \multicolumn{3}{|c|}{$\begin{array}{l}\text { Percepção dos discentes quanto à inserção de temática de } \\
\text { humanidades na grade curricular do curso de uma universidade } \\
\text { pública de Minas Gerais, } 2018 / 2019(\mathrm{n}=165)\end{array}$} \\
\hline Variáveis & $\mathbf{N}$ & $\%$ \\
\hline \multicolumn{3}{|c|}{ Seu curso tem matérias voltadas às humanidades? } \\
\hline Sim & 163 & 98,79 \\
\hline Não & 2 & 1,21 \\
\hline \multicolumn{3}{|c|}{ Quantas matérias de humanidades já cursou? } \\
\hline 1 & 1 & 0,61 \\
\hline 2 & 18 & 10,91 \\
\hline 3 & 26 & 15,76 \\
\hline 4 & 25 & 15,15 \\
\hline 5 ou mais & 92 & 55,76 \\
\hline Não respondeu & 3 & 1,81 \\
\hline \multicolumn{3}{|c|}{ Você gostava das disciplinas de humanidades? } \\
\hline Sim & 109 & 66,06 \\
\hline Não & 54 & 32,73 \\
\hline Não respondeu & 2 & 1,21 \\
\hline \multicolumn{3}{|c|}{$\begin{array}{l}\text { Quanto as disciplinas de humanidades exigem quando comparadas às } \\
\text { disciplinas técnicas? }\end{array}$} \\
\hline Exigem mais & 5 & 3,03 \\
\hline Exigem menos & 112 & 67,88 \\
\hline Exigem igualmente & 48 & 29,09 \\
\hline
\end{tabular}

Fonte: Elaborada pelos autores.

$3 \mid$\begin{tabular}{l|l} 
REVISTA BRASILEIRA DE EDUCAÇÃO MÉDICA \\
\hline 44 (4) : e167; 2020
\end{tabular} 
acerto foram sobre a indicação de via de parto em caso de gestação de baixo risco sem intercorrências intraparto (89,09\%). Desses itens, $82,61 \%$, $94,00 \%, 89,58 \%$ e $90,48 \%$ corresponderam aos acertos dos alunos do quarto, sexto, oitavo e décimo períodos, respectivamente. Outra questão com o maior índice de acerto referiu-se aos casos de gestação múltipla de dois fetos (69,70\%). Os alunos do quarto, sexto, oitavo e décimo períodos - 76,09\%, 56,00\%, 77,09 e 71,42\%, respectivamente - responderam que a via de parto depende da situação (Tabela 2).

\begin{tabular}{|c|c|c|c|c|c|c|c|c|c|c|}
\hline \multirow[t]{2}{*}{ Variáveis } & & $4^{\circ} \mathrm{P}$ & & $6^{\circ} \mathrm{P}$ & & $8^{\circ} \mathrm{P}$ & & $10^{\circ} \mathrm{P}$ & & Total \\
\hline & $n=46$ & $\%$ & $\mathbf{n}=\mathbf{5 0}$ & $\%$ & $n=48$ & $\%$ & $\mathrm{n}=\mathbf{2 1}$ & $\%$ & $n=165$ & $\%$ \\
\hline \multicolumn{11}{|c|}{ Que via de parto é indicada para gestantes de baixo risco sem intercorrências durante o trabalho de parto? } \\
\hline Vaginal* $^{*}$ & 38 & 82,61 & 47 & 94,00 & 43 & 89,58 & 19 & 90,48 & 147 & 89,09 \\
\hline Cesariana & --- & --- & $-\cdots$ & $-\cdots$ & $-\cdots$ & --- & --- & --- & $-\cdots$ & $-\cdots$ \\
\hline Depende da situação & 7 & 15,22 & 2 & 4,00 & 5 & 10,42 & 2 & 8,52 & 16 & 9,70 \\
\hline Não respondeu & 1 & 2,17 & 1 & 2,00 & --- & --- & ---- & ---- & 2 & 1,21 \\
\hline
\end{tabular}

Que via de parto é indicada para gestantes diabéticas, sem descompensação, macrossomia fetal ou outras complicações?

\begin{tabular}{|c|c|c|c|c|c|c|c|c|c|c|}
\hline Vaginal $^{*}$ & 6 & 13,04 & 16 & 32,00 & 14 & 29,17 & 6 & 28,57 & 42 & 25,45 \\
\hline Cesariana & 5 & 10,87 & 5 & 10,00 & 1 & 2,08 & 1 & 4,76 & 12 & 7,27 \\
\hline Depende da situação & 35 & 76,09 & 28 & 56,00 & 33 & 68,75 & 14 & 66,67 & 110 & 66,67 \\
\hline Não respondeu & ---- & --- & 1 & 2,00 & ---- & --- & ---- & --- & 1 & 0,61 \\
\hline
\end{tabular}

Que via de parto é indicada para gestação múltipla de dois fetos?

$\begin{array}{ccccccrrrrrr}\text { Vaginal } & 5 & 10,87 & 12 & 24,00 & 1 & 2,08 & 3 & 14,29 & 21 & 12,73 \\ \text { Cesariana } & 6 & 13,04 & 9 & 18,00 & 9 & 18,75 & 3 & 14,29 & 27 & 16,36 \\ \text { Depende da situação* } & 35 & 76,09 & 28 & 56,00 & 37 & 77,09 & 15 & 71,42 & 115 & 69,70 \\ \text { Não respondeu } & --- & ---- & 1 & 2,00 & 1 & 2,08 & -1,21\end{array}$

Que via de parto é indicada para gestantes apresentando pré-eclâmpsia?

\begin{tabular}{|c|c|c|c|c|c|c|c|c|c|c|}
\hline Vaginal $^{\star}$ & 3 & 6,52 & 3 & 6,00 & 23 & 47,92 & 1 & 4,76 & 30 & 18,18 \\
\hline Cesariana & 25 & 54,35 & 34 & 68,00 & 25 & 52,08 & 9 & 42,86 & 93 & 56,36 \\
\hline Depende da situação & 18 & 39,13 & 12 & 24,00 & ---- & ---- & 11 & 52,38 & 41 & 24,85 \\
\hline Não respondeu & --- & --- & 1 & 2,00 & --- & --- & --- & --- & 1 & 0,61 \\
\hline
\end{tabular}

Para a gestante em trabalho de parto progredindo dentro do esperado, a alimentação deve ser proibida ou restrita?

$\begin{array}{cccccccccccccc}\text { Sim } & 16 & 34,78 & 24 & 48,00 & 19 & 39,58 & 7 & 33,33 & 66 & 40,00 \\ \text { Não* }^{2} & 29 & 63,05 & 24 & 48,00 & 29 & 60,42 & 14 & 66,67 & 96 & 58,18\end{array}$

$\begin{array}{llllllll}\text { Não respondeu } & 1 & 2,17 & 2 & 4,00 & -\cdots & -\cdots\end{array}$

Para a gestante em trabalho de parto progredindo dentro do esperado, a hidratação deve ser proibida ou restrita?

\begin{tabular}{|c|c|c|c|c|c|c|c|c|c|c|}
\hline Sim & 2 & 4,35 & 6 & 12,00 & 5 & 10,42 & 3 & 14,29 & 16 & 9,70 \\
\hline Não* & 44 & 95,65 & 42 & 84,00 & 43 & 89,58 & 18 & 85,71 & 147 & 89,09 \\
\hline respondeu & ---- & ---- & 2 & 4,00 & ---- & ---- & ---- & ---- & 2 & 1,21 \\
\hline
\end{tabular}

Que via de parto proporciona uma recuperação mais rápida?

\begin{tabular}{|c|c|c|c|c|c|c|c|c|c|c|}
\hline Vaginal $^{\star}$ & 46 & 100,00 & 46 & 92,00 & 48 & 100,00 & 21 & 100,00 & 161 & 97,58 \\
\hline Cesariana & ---- & ---- & 2 & 4,00 & ---- & ---- & ---- & ---- & 2 & 1,21 \\
\hline Não respondeu & --- & ---- & 2 & 4,00 & ---- & ---- & --- & --- & 2 & 1,21 \\
\hline
\end{tabular}

A mulher em trabalho de parto possui direito a um acompanhante?

\begin{tabular}{|c|c|c|c|c|c|c|c|c|c|c|}
\hline $\operatorname{Sim}^{*}$ & 45 & 97,83 & 49 & 98,00 & 48 & 100,00 & 21 & 100,00 & 163 & 98,79 \\
\hline Não & ---- & ---- & ---- & ---- & ---- & ---- & ---- & ----- & ---- & ---- \\
\hline Não respondeu & 1 & 2,17 & 1 & 2,00 & ---- & ---- & --- & ---- & 2 & 1,21 \\
\hline
\end{tabular}

* Respostas corretas; $\mathrm{P}=$ período.

Fonte: Elaborada pelos autores. 
Com relação às questões com maiores índices de erro, destacamse as indicações de via de parto em caso de pré-eclâmpsia $(18,18 \%$ de acerto) e de via de parto em gestantes diabéticas (25,45\% de acerto). Com relação à primeira questão, a porcentagem de acerto separada por períodos foi de $6,52 \%$ para o quarto, $6,00 \%$ para o sexto, $47,92 \%$ para o oitavo e $4,76 \%$ para o décimo. Na segunda questão, responderam corretamente $13,04 \%$ do quarto período, $32,00 \%$ do sexto, $29,17 \%$ do oitavo e $28,57 \%$ do décimo (Tabela 2 ).

Houve também um percentual alto de acerto com relação ao direito de acompanhante da gestante durante o parto $(98,79 \%)$ e sobre que via de parto proporcionaria uma recuperação mais rápida (97,58\%) (Tabela 2).

Em outro grupo de questões, os participantes foram orientados a selecionar as opções que, segundo eles, estavam corretas com relação a diversos aspectos da parturição. As respostas relacionadas às posições mais indicadas para a primeira fase do trabalho de parto foram as seguintes: ginecológica $(89,09 \%)$ e cócoras $(72,12 \%)$. Separando as respostas por períodos, encontramos que $84,78 \%$ (ginecológica) e $73,91 \%$ (cócoras) para o quarto período, $82,00 \%$ (ginecológica) e $72,00 \%$ (cócoras) para o sexto período, $100 \%$ (ginecológica) e $60,42 \%$ (cócoras) para o oitavo período e $90,48 \%$ (ginecológica) e $95,24 \%$ (cócoras) para o décimo período. Obtiveram menor frequência as posições deambulando (13,33\%) e decúbito lateral direito $(4,85)$. Com relação à rotina realizada durante o parto vaginal, 56,97\% apontaram a realização do toque vaginal e 52,12\% indicaram a analgesia. Quanto aos procedimentos considerados violência obstétrica quando realizados sem indicação, as opções mais indicadas foram a episiotomia $(75,76 \%)$ e o uso de fórceps de rotina $(74,55 \%)$; e as respostas menos marcadas foram jejum (15,76\%) e tricotomia (16,36\%). É importante destacar que 9,09\% dos participantes indicaram a analgesia como violência obstétrica (Tabela 3).

Quando questionados sobre as vantagens do parto vaginal, os participantes marcaram em maior frequência a possibilidade de amamentação na primeira hora após o nascimento $(87,88 \%)$ e a liberação de hormônios que aumentam a satisfação da mulher (86,06\%). A questão menos assinalada foi a possibilidade de clampeamento tardio do cordão umbilical (10,30\%) (Tabela 3).

Com relação à opinião sobre diversos aspectos do parto vaginal, a maioria considera o parto vaginal como o mais seguro para a gestante (69,09\%) e para o bebê $(76,97 \%)$. Já para $75,15 \%$, o maior uso de tecnologias durante a assistência ao parto gera mais benefícios para a mãe e o bebê. A maioria $(85,45 \%)$ considera que o parto vaginal não traz problemas para a vida sexual da mulher. Apenas $1,82 \%$ considerou que a mulher não deve ter autonomia na decisão da via de parto. Já $98,79 \%$ acreditam que a humanização do acolhimento favorece o protagonismo da mulher durante o trabalho de parto (Tabela 4).

\begin{tabular}{|c|c|c|c|c|c|c|c|c|c|c|}
\hline \multicolumn{11}{|c|}{ Tabela 3} \\
\hline \multirow[t]{2}{*}{ Variáveis* } & \multirow{2}{*}{$\begin{array}{c}4^{\circ} \mathrm{P} \\
\mathrm{n}=46\end{array}$} & & \multirow{2}{*}{$\begin{array}{c}6^{\circ} \mathrm{P} \\
\mathrm{n}=50\end{array}$} & & \multirow{2}{*}{$\begin{array}{c}8^{\circ} \mathrm{P} \\
\mathrm{n}=48\end{array}$} & \multirow[b]{2}{*}{$\%$} & \multirow{2}{*}{$\begin{array}{c}10^{\circ} \mathrm{P} \\
\mathrm{n}=21\end{array}$} & \multicolumn{3}{|c|}{ Total } \\
\hline & & $\%$ & & $\%$ & & & & $\%$ & $n=165$ & $\%$ \\
\hline \multicolumn{11}{|c|}{ Quais posições são indicadas durante o trabalho de parto ativo? } \\
\hline Ginecológica & 39 & 84,78 & 41 & 82,00 & 48 & 100,00 & 19 & 90,48 & 147 & 89,09 \\
\hline Cócoras & 34 & 73,91 & 36 & 72,00 & 29 & 60,42 & 20 & 95,24 & 119 & 72,12 \\
\hline Em pé & 9 & 19,57 & 10 & 20,00 & 9 & 18,75 & 10 & 47,62 & 38 & 23,03 \\
\hline Deambulando & 5 & 10,87 & 3 & 6,00 & 5 & 10,42 & 9 & 42,86 & 22 & 13,33 \\
\hline Sentada & 7 & 15,22 & 10 & 20,00 & 10 & 20,83 & 6 & 28,57 & 33 & 20,00 \\
\hline Decúbito dorsal & 5 & 10,87 & 6 & 12,00 & 7 & 14,58 & 7 & 33,33 & 25 & 15,15 \\
\hline Decúbito lateral D & 3 & 6,52 & 1 & 2,00 & 1 & 2,08 & 3 & 14,29 & 8 & 4,85 \\
\hline \multicolumn{11}{|c|}{ Quais das seguintes intervenções são indicadas como rotina durante o parto vaginal? } \\
\hline Episiotomia & 4 & 8,70 & 6 & 12,00 & 10 & 20,83 & 4 & 19,05 & 24 & 14,55 \\
\hline Lavagem intestinal & 7 & 15,22 & 7 & 14,00 & 5 & 10,42 & ---- & --- & 19 & 11,52 \\
\hline Fórceps de rotina & 6 & 13,04 & 10 & 20,00 & 10 & 20,83 & ---- & ---- & 26 & 15,76 \\
\hline Indução & 10 & 21,74 & 7 & 14,00 & 8 & 16,67 & 1 & 4,76 & 26 & 15,76 \\
\hline Jejum & 7 & 15,22 & 8 & 16,00 & 10 & 20,83 & 5 & 23,81 & 30 & 18,18 \\
\hline Manobra Kristeller & 5 & 10,87 & 2 & 4,00 & 1 & 2,08 & ---- & ---- & 8 & 4,85 \\
\hline Amniotomia & 5 & 10,87 & 1 & 2,00 & 4 & 8,33 & 3 & 14,29 & 13 & 7,88 \\
\hline Tricotomia & 4 & 8,70 & 6 & 12,00 & 11 & 22,92 & 4 & 19,05 & 25 & 15,15 \\
\hline Toque & 25 & 54,35 & 25 & 50,00 & 27 & 56,25 & 17 & 80,95 & 94 & 56,97 \\
\hline Analgesia & 20 & 43,48 & 23 & 46,00 & 30 & 62,5 & 13 & 61,9 & 86 & 52,12 \\
\hline Nenhuma & 6 & 13,04 & 8 & 16,00 & 4 & 8,33 & 4 & 19,05 & 22 & 13,33 \\
\hline
\end{tabular}

\begin{tabular}{l|l}
\hline REVISTA BRASILEIRA DE EDUCAÇÃO MÉDICA \\
\hline 44 (4) : e167; 2020
\end{tabular} 


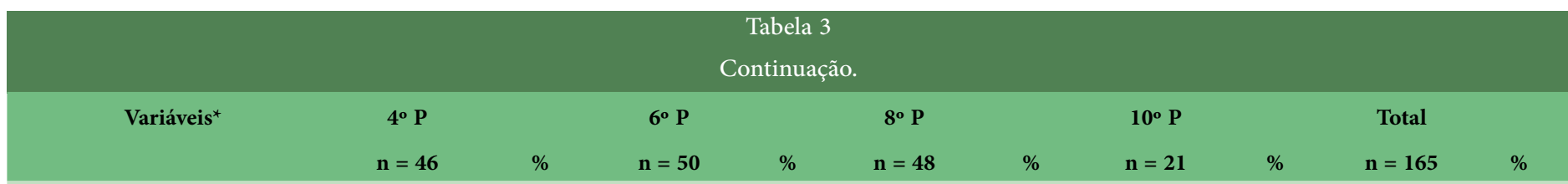

Quais das seguintes intervenções são consideradas violência obstétrica quando realizadas sem indicação?

\begin{tabular}{|c|c|c|c|c|c|c|c|c|c|c|}
\hline Episiotomia & 35 & 76,09 & 35 & 70,00 & 35 & 72,92 & 20 & 95,24 & 125 & 75,76 \\
\hline Lavagem intestinal & 15 & 32,61 & 10 & 20,00 & 12 & 25,00 & 8 & 38,10 & 45 & 27,27 \\
\hline Fórceps de rotina & 31 & 67,39 & 39 & 78,00 & 33 & 68,75 & 20 & 95,24 & 123 & 74,55 \\
\hline Indução & 26 & 56,52 & 17 & 34,00 & 22 & 45,83 & 11 & 52,38 & 76 & 46,06 \\
\hline Jejum & 11 & 23,91 & 8 & 16,00 & 3 & 6,25 & 4 & 19,05 & 26 & 15,76 \\
\hline Manobra Kristeller & 11 & 23,91 & 9 & 18,00 & 20 & 41,67 & 17 & 80,95 & 57 & 34,55 \\
\hline Amniotomia & 10 & 21,74 & 5 & 10,00 & 19 & 39,58 & 13 & 61,90 & 47 & 28,48 \\
\hline Tricotomia & 12 & 26,09 & 4 & 8,00 & 7 & 14,58 & 4 & 19,05 & 27 & 16,36 \\
\hline Toque & 8 & 17,39 & 11 & 22,00 & 5 & 10,42 & 5 & 23,81 & 29 & 17,58 \\
\hline Analgesia & 7 & 15,22 & 3 & 6,00 & 2 & 4,17 & 3 & 14,29 & 15 & 9,09 \\
\hline Nenhuma & ---- & ---- & 1 & 2,00 & 2 & 4,17 & ---- & ---- & 3 & 1,82 \\
\hline
\end{tabular}

Quais condições abaixo são favorecidas pelo parto vaginal?

\begin{tabular}{|c|c|c|c|c|c|c|c|c|c|c|}
\hline Amamentação & 43 & 93,48 & 38 & 76,00 & 44 & 91,67 & 20 & 95,24 & 145 & 87,88 \\
\hline Ruptura uterina & 2 & 4,35 & 2 & 4,00 & 2 & 4,17 & 2 & 9,52 & 8 & 4,85 \\
\hline Contato & 36 & 78,26 & 40 & 80,00 & 42 & 87,5 & 21 & 100 & 139 & 84,24 \\
\hline Liberação & 41 & 89,13 & 41 & 82,00 & 42 & 87,5 & 18 & 85,71 & 142 & 86,06 \\
\hline Mortalidade materna & 3 & 6,52 & 1 & 2,00 & 1 & 2,08 & --- & ---- & 5 & 3,03 \\
\hline Clampeamento tardio & 6 & 13,04 & 3 & 6,00 & 2 & 4,17 & 6 & 28,57 & 17 & 10,30 \\
\hline Morbidade fetal & 1 & 2,17 & ---- & ---- & ---- & ---- & ---- & ---- & 1 & 0,61 \\
\hline Desconforto respiratório & 34 & 73,91 & 28 & 56,00 & 26 & 54,17 & 19 & 90,48 & 107 & 64,85 \\
\hline
\end{tabular}

${ }^{*}$ Houve mais de uma resposta por questão. $\mathrm{P}=$ período.

Fonte: Elaborada pelos autores.

\section{Tabela 4}

Opinião dos participantes sobre via de parto - universidade pública de Minas Gerais - 2018/2019 (n = 165)

$\begin{array}{llllll}\text { Variáveis } & 4^{\circ} \mathrm{P} & 6^{\circ} \mathrm{P} & 8^{\circ} \mathrm{P} & 10^{\circ} \mathrm{P} & \text { Total }\end{array}$

$\begin{array}{lllllllll}\mathrm{n}=46 & \mathrm{n}=50 & \% & \mathrm{n}=48 & \% & \mathrm{n}=21 & \% & \mathrm{n}=165 & \%\end{array}$

Qual via de parto você julga mais segura para a gestante?

$\begin{array}{ccccccccccc}\text { Vaginal } & 42 & 91,30 & 46 & 92,00 & 6 & 12,50 & 20 & 95,24 & 114 & 69,09 \\ \text { Cesariana } & 4 & 8,70 & 3 & 6,00 & 42 & 87,50 & 1 & 4,76 & 50 & 30,30 \\ \text { Não respondeu } & ---- & ---- & 1 & 2,00 & ---- & ---- & ---- & --- & 1 & 0,61\end{array}$

Qual via de parto você julga mais segura para o bebê?

$\begin{array}{ccccccccccc}\text { Vaginal } & 37 & 80,43 & 39 & 78,00 & 34 & 70,83 & 17 & 80,95 & 127 & 76,97 \\ \text { Cesariana } & 9 & 19,57 & 10 & 20,00 & 14 & 29,17 & 4 & 19,05 & 37 & 22,42 \\ \text { Não respondeu } & ---- & ---- & 1 & 2,00 & ---- & ---- & --- & --- & 1 & 0,61\end{array}$

Você considera que o uso de mais tecnologias durante o parto o torna mais benéfico para a mãe e o bebê?

$\begin{array}{lccccccccccc}\text { Sim } & 35 & 76,09 & 37 & 74,00 & 36 & 75,00 & 16 & 76,19 & 124 & 75,15 \\ \text { Não } & 11 & 23,91 & 12 & 24,00 & 12 & 25,00 & 4 & 19,05 & 39 & 23,64 \\ \text { o respondeu } & ---- & ---- & 1 & 2,00 & ---- & ---- & 1 & 4,76 & 2 & 1,21\end{array}$




\begin{tabular}{|c|c|c|c|c|c|c|c|c|c|c|}
\hline \multicolumn{11}{|c|}{$\begin{array}{cc}\text { Tabela } 4 \\
\text { Continuação. }\end{array}$} \\
\hline \multirow[t]{2}{*}{ Variáveis } & \multicolumn{3}{|c|}{$4^{\circ} \mathrm{P}$} & \multirow{2}{*}{$\begin{array}{l}6^{\circ} \mathrm{P} \\
\%\end{array}$} & \multirow[b]{2}{*}{$\mathrm{n}=48$} & \multirow{2}{*}{$\begin{array}{l}8^{\circ} \mathrm{P} \\
\%\end{array}$} & \multirow{2}{*}{\multicolumn{2}{|c|}{$\begin{array}{l}10^{\circ} \mathrm{P} \\
\%\end{array}$}} & \multirow[b]{2}{*}{$\mathrm{n}=165$} & \multirow{2}{*}{$\begin{array}{c}\text { Total } \\
\%\end{array}$} \\
\hline & $\mathrm{n}=46$ & $\%$ & $\mathrm{n}=50$ & & & & & & & \\
\hline \multicolumn{11}{|c|}{ Você considera que o parto vaginal traz prejuízos à vida sexual da mulher? } \\
\hline Sim & 5 & 10,87 & 7 & 14,00 & 7 & 14,58 & 4 & 19,05 & 23 & 13,94 \\
\hline Não & 41 & 89,13 & 42 & 84,00 & 41 & 85,42 & 17 & 80,95 & 141 & 85,45 \\
\hline Não respondeu & --- & ---- & 1 & 2,00 & $---\cdot$ & ---- & --- & $---\cdot$ & 1 & 0,61 \\
\hline \multicolumn{11}{|c|}{ Você considera que a mulher deve ter autonomia na decisão da via de parto? } \\
\hline Sim & 46 & 100,00 & 47 & 94,00 & 46 & 95,84 & 21 & 100,00 & 160 & 96,97 \\
\hline Não & --- & --- & 2 & 4,00 & 1 & 2,08 & --- & --- & 3 & 1,82 \\
\hline Não respondeu & --- & --- & 1 & 2,00 & 1 & 2,08 & --- & --- & 2 & 1,21 \\
\hline \multicolumn{11}{|c|}{ Você considera que o tipo de acolhimento prestado à gestante influencia no andamento do trabalho de parto? } \\
\hline Sim & 46 & 100,00 & 49 & 98,00 & 48 & 100,00 & 21 & 100,00 & 164 & 99,39 \\
\hline Não & --- & --- & --- & --- & $-\cdots$ & --- & --- & --- & --- & --- \\
\hline Não respondeu & --- & --- & 1 & 2,00 & --- & --- & ---- & ---- & 1 & 0,61 \\
\hline
\end{tabular}

Você acredita que a humanização do acolhimento incentiva o protagonismo da mulher durante o trabalho de parto?

\begin{tabular}{|c|c|c|c|c|c|c|c|c|c|c|}
\hline Sim & 46 & 100,00 & 48 & 96,00 & 48 & 100,00 & 21 & 100,00 & 163 & 98,79 \\
\hline Não & ---- & ---- & 1 & 2,00 & ---- & ---- & ---- & ---- & 1 & 0,61 \\
\hline respondeu & ---- & ---- & 1 & 2,00 & ---- & ---- & ---- & --- & 1 & 0,61 \\
\hline
\end{tabular}

Considerando os conhecimentos adquiridos durante a graduação, qual via de parto você escolheria para o nascimento de um filho seu cuja gestação foi tranquila e sem intercorrências?

\begin{tabular}{|c|c|c|c|c|c|c|c|c|c|c|}
\hline Vaginal & 37 & 80,43 & 43 & 86,00 & 43 & 89,58 & 14 & 66,67 & 137 & 83,03 \\
\hline Cesariana & 9 & 19,57 & 6 & 12,00 & 5 & 10,42 & 7 & 33,33 & 27 & 16,36 \\
\hline Não respondeu & ---- & --- & 1 & 2,00 & --- & --- & ---- & --- & 1 & 0,61 \\
\hline
\end{tabular}

Você considera que o medo da dor do parto vaginal é um empecilho para o incentivo dele?

\begin{tabular}{|c|c|c|c|c|c|c|c|c|c|c|}
\hline Sim & 44 & 95,65 & 40 & 80,00 & 42 & 87,50 & 20 & 95,24 & 146 & 88,48 \\
\hline Não & 2 & 4,35 & 9 & 18,00 & 6 & 12,50 & 1 & 4,76 & 18 & 10,91 \\
\hline respondeu & ---- & ---- & 1 & 2,00 & ---- & ---- & --- & ---- & 1 & 0,61 \\
\hline
\end{tabular}

Você considera que o medo da dor do parto vaginal é um incentivo para a realização da cesariana?

\begin{tabular}{|c|c|c|c|c|c|c|c|c|c|c|}
\hline Sim & 44 & 95,65 & 41 & 82,00 & 34 & 70,83 & 20 & 95,24 & 139 & 84,24 \\
\hline Não & 2 & 4,35 & 8 & 16,00 & 14 & 29,17 & 1 & 4,76 & 25 & 15,15 \\
\hline respondeu & --- & ---- & 1 & 2,00 & --- & ---- & -.-- & --.- & 1 & 0,61 \\
\hline
\end{tabular}

Você indicaria uma cesariana motivado (a) pela comodidade de escolha do dia e horário para realização dela para uma gestante de baixo risco e sem intercorrências?

$\begin{array}{lllllllllll}\text { Sim } & 7 & 15,22 & 12 & 24,00 & 13 & 27,08 & 4 & 19,05 & 36 & 21,82 \\ \text { Não } & 39 & 84,78 & 37 & 74,00 & 35 & 72,92 & 17 & 80,95 & 128 & 77,58\end{array}$

Não respondeu

$\begin{array}{llll}--- & --- & 1 & 2,00\end{array}$

$---$

--- ----

$----$

1

0,61

Você considera necessário que o profissional durante a assistência ao trabalho de parto informe todos os procedimentos à gestante?

\begin{tabular}{|c|c|c|c|c|c|c|c|c|c|c|}
\hline Sim & 45 & 97,83 & 49 & 98,00 & 47 & 97,92 & 21 & 100,00 & 162 & 98,18 \\
\hline Não & 1 & 2,17 & ---- & ---- & 1 & 2,08 & ---- & ---- & 2 & 1,21 \\
\hline respondeu & --.- & --- & 1 & 2,00 & --- & ---- & --- & --- & 1 & 0,61 \\
\hline
\end{tabular}

Você considera que a via de parto pode ter benefícios psicológicos para a mãe e melhorar a relação mãe-bebê no puerpério?

\begin{tabular}{|c|c|c|c|c|c|c|c|c|c|c|}
\hline Sim & 46 & 100,00 & 48 & 96,00 & 48 & 100,00 & 21 & 100,00 & 163 & 98,79 \\
\hline Não & -.-- & --- & 1 & 2,00 & ---- & ---- & ---- & --- & 1 & 0,61 \\
\hline respondeu & ---- & --- & 1 & 2,00 & ---- & ---- & ---- & ---- & 1 & 0,61 \\
\hline
\end{tabular}

$\mathrm{P}=$ período.

Fonte: Elaborada pelos autores. 
Em relação à via de parto para o nascimento dos filhos dos participantes no caso de uma gestação sem intercorrências, a via vaginal foi a opção mais apontada (83,03\%). Para $84,24 \%$, a dor do parto é um incentivo à realização da cirurgia cesariana. Com relação à indicação de parto via cesárea pela comodidade de escolha do dia e horário de sua realização, a maioria $(77,58 \%)$ respondeu que não indicaria (Tabela 4).

\section{DISCUSSÃO}

Quanto ao perfil dos participantes, os resultados indicam a maior frequência de discentes na faixa etária de 18 a 22 anos, do sexo feminino, solteiros e sem filhos. Os achados sociodemográficos encontrados condizem com os resultados de outros estudos com população semelhante. Em uma investigação realizada na Universidade do Estado de Mato Grosso (Unemat), identificou-se o predomínio do sexo feminino (54\%), na faixa etária de 18 a 23 anos (82\%), entre os discentes do curso de Medicina participantes do estudo $^{11}$. Em outra pesquisa realizada na Pontifícia da Universidade Católica de Goiás (PUC Goiás), verificou-se o predomínio de estudantes do sexo feminino $(68,1 \%)$ com média de idade de $24,3 \operatorname{anos}^{12}$.

Os acadêmicos foram questionados sobre a presença de disciplinas de humanidades na matriz curricular. Essas disciplinas pertencem a uma área que engloba os conhecimentos de filosofia, antropologia, psicologia, sociologia, história, política, artes e ética, voltadas para o âmbito da saúde e do cuidado ${ }^{13}$. O ensino das humanidades nos cursos da área da saúde é visto como essencial por desenvolver uma maior compreensão do sofrimento humano e das manifestações que acompanham o adoecimento, assim como possibilita o conhecimento de habilidades de comunicação e a construção de vínculos essenciais para a prestação de um cuidado de qualidade ${ }^{14}$. No curso de Medicina da instituição em estudo, as disciplinas tidas como humanidades são ministradas entre o primeiro e o quinto período, concentrando-se, portanto, no ciclo básico.

Todos os alunos questionados sobre a quantidade de disciplinas já cursadas de humanidades já haviam feito todas (alunos do sexto, oitavo e décimo períodos) ou já haviam realizado a maioria (alunos do quarto período). No entanto, houve discordância de respostas com relação ao número de disciplinas já cursadas, o que pode ser relacionado à percepção subjetiva dos discentes quanto ao conceito de humanidades, classificando de forma variada as determinadas disciplinas. Para os participantes, as disciplinas menos técnicas exigiam menos do que as técnicas ou estavam no mesmo patamar. Em um estudo realizado na Universidade de São Paulo (USP) com estudantes de Medicina, em que se abordaram assuntos relacionados a humanidades, concluiu-se que é necessária uma maior abordagem da humanização na matriz curricular para a formação de um profissional que não seja unicamente técnico e possa, durante a graduação e também futuramente, desenvolver uma relação mais humana com os pacientes ${ }^{15}$.

Antes da discussão dos itens referentes ao conhecimento dos estudantes de Medicina sobre alguns aspectos da via de parto e outros que englobam a área da ginecologia e obstetrícia, é importante mencionar que, na universidade em questão, as disciplinas de ginecologia e obstetrícia começam a ser ofertadas a partir do sétimo período e são abordadas até o último semestre da faculdade.

Quase a totalidade dos participantes assinalou que a presença de acompanhante durante o parto é um direito da gestante. De acordo com a Lei Federal no 11.108, de 7 de abril de 2005, os serviços de saúde do
Sistema Único de Saúde (SUS), da rede privada ou da conveniada são obrigados a permitir a presença de acompanhante durante o trabalho de parto, o parto e o pós-parto imediato ${ }^{16}$. A presença do acompanhante durante o parto traz benefícios como o apoio emocional que torna a mulher mais confiante, permitindo que o nascimento ocorra de forma mais fisiológica, além de reduzir a dor, o nervosismo e a sensação de solidão. O acompanhante facilita a explicação dos procedimentos para as parturientes, visto que, por conta do nervosismo, muitas vezes elas não entendem o que está sendo explicado pela equipe ${ }^{17}$.

$\mathrm{Na}$ questão sobre as posições mais indicadas para a parturiente durante a primeira fase de trabalho de parto, as respostas mais frequentes foram: ginecológica e cócoras. Já as menos frequentes foram: deambulando e decúbito lateral direito. Não houve diferenças entre as respostas dos alunos dos períodos analisados. Para todos os períodos questionados, as duas respostas citadas anteriormente foram as mais prevalentes. Ressaltase que as disciplinas que abordam a temática se iniciam a partir do sétimo período, demonstrando que, mesmo com o avançar do curso e com o início de disciplinas que tratam do assunto, a percepção do estudante sobre as posições não se altera significativamente. Há sim um aumento nas respostas das posições de pé e deambulando entre os alunos do décimo período, no entanto ainda representam menos da metade das respostas. A OMS recomenda que a gestante de baixo risco seja encorajada a adotar posturas verticais e mobilidade durante a primeira fase do trabalho de parto. Informar à mulher as vantagens dessas posturas faz parte do cuidado intraparto, deixando-a livre para escolher a postura em que se sinta mais confortável ${ }^{18}$.

A maioria dos participantes desta pesquisa respondeu que a via vaginal proporciona recuperação mais rápida da gestante. A OMS recomenda que, após um parto vaginal sem complicações, com mãe e bebê saudáveis, estes devem ser observados e cuidados em serviço de saúde por, no mínimo, 24 horas depois do nascimento ${ }^{18}$. A média de tempo de internação após uma cesárea varia de três a quatro dias, porém mulheres afebris e sem complicações podem receber alta mais precocemente ${ }^{19}$. Por conta da recuperação mais rápida, as mulheres preferem o parto pela via vaginal ${ }^{20}$.

Nas questões sobre as indicações de via de parto e outros aspectos relacionados a ele, os discentes apresentaram maior índice de acerto nas variáveis sobre a indicação de via de parto em caso de gestação de baixo risco e sem intercorrências intraparto e gestação múltipla de dois fetos. Esse índice de acerto foi maior entre os estudantes do sexto período e não entre os do oitavo e décimo períodos. Esse resultado não era esperado pelo fato de o início do conteúdo sobre ginecologia e obstetrícia ocorrer no sétimo período. A via de parto de escolha para gestantes de baixo risco e sem intercorrência é a via vaginal. A cesárea só deve ser realizada nos casos em que o parto vaginal gere risco à vida da mãe e/ou do bebê $\hat{e}^{21}$. Em gestações com dois gemelares, a via vaginal deverá ser escolhida apenas quando o primeiro gemelar estiver em posição cefálica. Contudo, é importante verificar também se ambos estão em posição cefálica, mesmo com peso inferior a 1.500 gramas e monoamnióticos. Deve-se confirmar ainda se não há evidência ultrassonográfica de encravamento, cordão umbilical entrelaçado ou síndrome de transfusor-transfundido. Já o parto cesáreo é indicado sempre que o primeiro gemelar está em posição não cefálica. Vale mencionar também que há muita controvérsia na literatura quanto às recomendações em relação às gestações gemelares, isto é, a corionicidade, o risco de complicações maternas e fetais e a 
apresentação dos fetos devem ser levados em consideração, com conduta individualizada para cada caso ${ }^{22}$.

Para os alunos do quarto, sexto e oitavo períodos, a via de escolha para gestante com pré-eclâmpsia, sem outras complicações, seria a cirurgia cesariana, e, para os alunos do décimo período, isso depende da situação. A diferença de respostas evidencia uma maior criticidade na escolha dos estudantes do décimo período provavelmente por estarem mais avançados no curso. A via de parto recomendada em gestantes com pré-eclâmpsia, sem outras complicações, em geral, é a via vaginal por acarretar maiores benefícios maternos. O parto cesáreo só deverá ser tentado nessas gestantes se houver indicação específica, uma vez que o risco de complicações maternas aumenta com a realização de cirurgia cesariana. Em gestantes com pré-eclâmpsia grave, há maior risco para o desenvolvimento de eventos hemorrágicos, picos hipertensivos, aumento do tempo de hospitalização e risco elevado de infecções ${ }^{23}$.

A pré-eclâmpsia é a síndrome específica da gestação responsável pela maior taxa de mortalidade materna. Além disso, as suas manifestações sistêmicas são responsáveis por danos em diversos órgãos, como cérebro, rins e fígado. Uma assistência adequada proporciona redução de sua incidência e da mortalidade das gestantes, uma vez que é considerada uma causa de morte evitável, reversível e prevenível ${ }^{24}$. A alta incidência de erro nessa questão é relevante e preocupante por causa dos desfechos negativos que podem ocorrer na gestação, no parto, no puerpério e no restante da vida da parturiente.

Para os estudantes de todos os períodos avaliados, a escolha da via de parto para gestantes diabéticas sem outras complicações, sem descompensação e com o bebê sem macrossomia depende da situação. De forma geral, nessas condições, a via de parto vaginal deve ser a preconizada. No caso de gestantes diabéticas, às vezes é necessário que o parto seja induzido mais cedo para evitar macrossomia, se houver descompensação no controle glicêmico ou complicações vasculares, porém a via de parto indicada é a vaginal. A cirurgia cesariana apenas deve ser indicada em caso de macrossomia quando há discordância cefalopélvica ${ }^{25}$.

A maioria dos estudantes avaliados considera o parto vaginal como o mais seguro para a gestante e o bebê. Mais da metade deles considera que o maior uso de tecnologias durante a assistência ao parto gera mais benefícios para a mãe e o bebê. $\mathrm{O}$ momento do parto é marcante para a mulher e pode oferecer lembranças positivas ou negativas, o que dependerá da vivência. Por isso, a assistência fornecida deve ser realizada de forma sensível às necessidades da mulher e permitir-lhe uma posição de autonomia nesse processo. As técnicas, os procedimentos e as orientações dadas à mulher durante o trabalho de parto devem ter como finalidade aproximá-lo de um processo natural e saudável. $\mathrm{O}$ uso de tecnologias duras, instrumentais e procedimentos específicos e apenas realizados por profissionais capacitados (como uso de fórceps e episiotomia) tem ganhado destaque, principalmente durante a segunda fase do trabalho de parto, de forma, muitas vezes, desnecessária, o que pode colocar em risco a saúde da mulher e do bebê; tais riscos poderiam ser evitados se a fisiologia do parto fosse respeitada ${ }^{26}$.

Nesse contexto, para mudar esse cenário de uso de tecnologias leves, relacionadas à relação interpessoal entre profissionais e usuários, é imprescindível que haja um esforço dos profissionais médicos no fortalecimento de aspectos fundamentais como acolhimento, criação de vínculos e autonomia da mulher. $\mathrm{O}$ movimento de humanização do parto luta nessa direção, com o objetivo de reduzir as intervenções desnecessárias e permitir o nascimento o mais próximo possível do fisiológico e natural, sem colocar a mulher ou o bebê em risco, intervindo caso haja necessidade, sempre de acordo com as evidências científicas ${ }^{26}$.

Com relação aos procedimentos considerados violência obstétrica quando realizados sem indicação, os discentes apontaram a episiotomia e o uso de fórceps de rotina. $\mathrm{O}$ jejum e a tricotomia obtiveram os menores índices. De maneira geral, o décimo período da Medicina obteve maior número de acertos ao indicar quais procedimentos são considerados violência obstétrica, sugerindo mudança na percepção de acordo com o avançar das disciplinas. Destaca-se que 9,09\% dos acadêmicos indicaram analgesia como violência obstétrica. Em relação a esse tema, todas as opções no instrumento utilizado para a coleta de dados são consideradas violência obstétrica se realizadas de rotina e sem indicação, exceto a analgesia. Violência obstétrica também está associada à recusa de prestação do serviço de saúde à gestante, à demora na realização da assistência, à não aplicação de analgesia, a procedimentos feitos de forma coerciva ou sem o consentimento da mulher e a maus-tratos psicológicos, verbais ou físicos ${ }^{27}$.

Entre os discentes que participaram desta pesquisa, apenas $1,82 \%$ considera que a mulher não deve ter autonomia na decisão da via de parto. Para a maioria, o profissional de saúde deve informar à mulher todos os procedimentos que serão realizados durante o trabalho de parto. A violência obstétrica engloba também o uso excessivo de medicações e intervenções durante o parto, além de práticas desagradáveis e dolorosas para as mulheres, sem evidência científica para sua realização. Entre as práticas consideradas violência obstétrica, destacam-se episiotomias de rotina, tricotomia dos pelos pubianos, manobra de Kristeller, exames de toque feitos repetidamente, lavagem intestinal, amniotomia e uso de fórceps de rotina ${ }^{28-31}$. A prática da episiotomia de rotina aumenta o risco de laceração perineal, hemorragia e infecções, além de não reduzir as complicações em longo prazo, como dor e incontinências fecal e urinária.

A manobra de Kristeller está ligada às internações em unidades de terapia intensiva neonatal para o bebê e lacerações perineais para a mãe. Restrições hídricas e alimentares prolongadas da gestante podem levar a um desconforto da mulher. As parturientes devem ter liberdade para ingerir alimentos leves ou líquidos durante o trabalho de parto. Para 40\% dos participantes da pesquisa, a alimentação deve ser proibida ou restrita, e $89,09 \%$ consideram que a hidratação deve ser autorizada. A amniotomia para a realização de trabalho de parto mais rápido aumenta a chance de cesariana ${ }^{30}$.

Ainda sobre violência obstétrica, considera-se o toque vaginal um tipo de violência quando é executado com uma frequência elevada e por vários profissionais em curtos períodos, uma vez que se trata de um procedimento doloroso e desagradável, que pode causar edema e desconforto na vulva, e ser prejudicial para a evolução do parto $^{32}$. A OMS não recomenda a tricotomia e indica o toque vaginal em intervalos de quatro horas durante a primeira fase do trabalho de parto em gestantes de baixo risco. Alimentos e líquidos por via oral são permitidos para as gestantes de baixo risco durante todo o trabalho de parto. A amniotomia de rotina ou sua associação com ocitocina para acelerar o trabalho de parto ou prevenir atraso do parto, em fases precoces deste, não é recomendada ${ }^{18}$.

Já sobre as vantagens do parto vaginal, os participantes apontaram com maior frequência a possibilidade de amamentação na primeira hora após o nascimento e a liberação de hormônios que aumentam a

REVISTA BRASILEIRA DE EDUCAÇÃO MÉDICA

9 44 (4) : e167; 2020 
satisfação da mulher. Quase todos os estudantes acreditam que o parto vaginal proporciona benefícios psicológicos para a mãe e melhora a relação mãe-bebê no puerpério. A questão que obteve menos respostas referiu-se à possibilidade de clampeamento tardio do cordão umbilical. Esse tipo de clampeamento ocorre após um a três minutos depois do nascimento do bebê ou até que se interrompam as pulsações do cordão ${ }^{33}$. O clampeamento tardio pode ser realizado na cesárea, porém a transfusão de sangue para o bebê é menor quando comparada com o parto vaginal ${ }^{34}$.

É importante ressaltar também que a OMS recomenda que os recém-nascidos sem complicações devem ser mantidos em contato pele a pele com as mães durante a primeira hora de vida, como forma de evitar hipotermia e estimular a amamentação. Também recomenda que a amamentação deve ser realizada o mais rápido possível, assim que a mãe e o bebê apresentarem condições clínicas estáveis ${ }^{20}$. Mulheres que passaram por cesárea têm menor probabilidade de conseguir amamentar nas primeiras horas após o nascimento ${ }^{19}$. Esse atraso na amamentação pode ocorrer pela limitação de movimentos imposta pela cirurgia e pela necessidade de cuidados pós-operatórios. Além disso, deve-se considerar que essa prática aumenta o tempo cirúrgico da cesárea, e, com isso, há mais riscos de infecção materna ${ }^{35}$.

A ocitocina e seu padrão natural de liberação durante o parto e a amamentação tornam cada trabalho de parto único e são responsáveis pela criação do laço afetivo entre a mãe e a bebê. A ocitocina também transforma a percepção dolorosa das contrações do parto vaginal em uma percepção mais positiva. Além disso, ela é importante na prevenção de hemorragia no pós-parto imediato ${ }^{36}$.

Para a maioria dos respondentes, o parto vaginal não resulta em problemas para a vida sexual da mulher. A retomada da vida sexual é geralmente mais rápida em mulheres que tiveram parto vaginal sem episiotomia quando comparadas àquelas que tiveram parto vaginal com episiotomia, parto vaginal instrumentalizado, cesariana eletiva ou de emergência. No parto vaginal, ocorre o alongamento do corpo perineal, o que não afeta a função do assoalho pélvico. Apesar de o parto vaginal estar mais relacionado com traumatismos perineais, a literatura sugere que mulheres que passaram por cesárea são mais propensas a se queixar de dispareunia. Entretanto, no geral, tal queixa tende a diminuir num período que varia de seis a 12 semanas após o parto, e, 12 meses depois do parto, parece não haver diferença na função sexual, independentemente do tipo de parto ${ }^{37}$.

Os resultados mostraram que, para a maior parte dos estudantes avaliados, o tipo de acolhimento recebido durante o trabalho de parto influencia no andamento dele. Segundo a OMS, os cuidados prestados a toda gestante devem ser respeitosos, de modo a garantir a dignidade, privacidade e confidencialidade para evitar maus-tratos, assegurar a liberdade de escolha informada da gestante e fornecer apoio contínuo durante o trabalho de parto e os partos ${ }^{18}$. Da mesma forma, quase todos os participantes pontaram que a humanização desse acolhimento favorece o protagonismo da mulher durante o trabalho de parto. Em estudo realizado com acadêmicos de Medicina do quinto e sexto anos, verificou-se que $22,0 \%$ deles identificaram na prática uma falta de autonomia das gestantes na escolha dos procedimentos e na via de parto a serem realizados ${ }^{38}$.

A via vaginal foi o aspecto mais mencionado no que concerne ao método para o nascimento dos seus filhos em caso de uma gestação sem intercorrências. Um estudo realizado com médicos ginecologistas e obstetras sobre a preferência da via de parto verificou que $44,0 \%$ dos homens recomendavam o parto vaginal para as esposas; já as mulheres $(37,8 \%)$ recomendavam a escolha pela via vaginal para si mesmas. Sobre a indicação da via de parto para as filhas, $43,5 \%$ responderam que indicariam o parto normal, $32,3 \%$ mencionaram o cesáreo e $24,2 \%$ não fariam indicações. A maioria desses profissionais teve seus filhos por meio de parto cesáreo $(79,2 \%)$, e essa taxa foi de $75,5 \%$ de cesáreas no primeiro filho e $83,2 \%$ no segundo, contrastando com a recomendação sobre o tipo de via que esses profissionais indicariam para as esposas ou que elas indicariam para si mesmas ${ }^{39}$.

A dor do parto é um empecilho para o incentivo à via vaginal para a maioria desse grupo de alunos, pois grande parte deles considera a dor um estímulo à realização da cesariana. A OMS recomenda intervenções educacionais com discussões sobre dor do parto e técnicas farmacológicas e não farmacológicas para o controle da dor (seus riscos e benefícios), como intervenções não clínicas para reduzir as cesarianas desnecessárias, e o grau de evidência dessa recomendação varia de baixo a moderado ${ }^{40}$. O medo da dor do parto vaginal foi uma das principais razões exposta pelas gestantes para a escolha da cirurgia cesariana ${ }^{20,41}$.

A maioria dos estudantes avaliados não indicaria a cesárea pela comodidade de escolha do dia e horário da sua realização. Alguns profissionais de saúde justificam as cesáreas por valorizarem a conveniência de agendamento e o menor tempo de comprometimento com a assistência tanto para si como para as mulheres, quando comparadas com o parto vaginal ${ }^{40}$.

Quando a cesárea é indicada por um médico, recomenda-se tal indicação seja confirmada por uma segunda opinião, com qualificação igual ou superior à do primeiro, trabalhando no mesmo serviço. Essa recomendação tem como objetivo reduzir as cesáreas desnecessárias e se baseia no fato de não haver entre os profissionais de saúde um consenso claro sobre o que é ou não uma indicação clínica para a cesárea, principalmente entre profissionais menos experientes; dessa forma, haveria troca de opiniões com profissionais mais experientes ${ }^{40}$.

Destaca-se como limitação do estudo a aplicação das mesmas questões sobre aspectos técnicos das vias de parto para períodos que não haviam tido as disciplinas de ginecologia e obstetrícia no curso, o que pode ter aumentado a taxa de erros nas respostas. Também não foram comparadas estatisticamente as respostas dos acadêmicos entre os períodos do curso, no intuito de avaliar se houve melhora de desempenho dos alunos com o avançar do curso e do acúmulo de conhecimento.

Com isso, são necessários novos estudos para avaliar o impacto que a matriz curricular dos cursos de Medicina tem sobre o perfil do egresso, para que se fortaleçam aspectos que valorizem as habilidades humanísticas desses profissionais, e, com isso, reverter o cenário de epidemia de cesáreas, além de favorecer a assistência humanizada às mulheres.

\section{CONCLUSÕES}

$\mathrm{Na}$ amostra estudada, o perfil é predominantemente feminino, sem filhos e na faixa etária de 18 a 22 anos. Observaram-se discrepâncias entre as perguntas referentes ao conhecimento técnico sobre a via de parto entre os estudantes dos diferentes períodos abordados. Para a maior parte dessas perguntas, os acertos foram maiores nos períodos mais avançados do curso, no entanto algumas questões seguiram com índices de acertos similares quando se compararam os períodos. Observou-se que, nas questões não técnicas, essas discrepâncias foram menores.

\footnotetext{
$10 \mid$\begin{tabular}{l|l} 
REVISTA BRASILEIRA DE EDUCAÇÃO MÉDICA \\
\hline $4(4):$ e $167 ; 2020$
\end{tabular}
} 


\section{REFERÊNCIAS}

1. Betrán AP, Ye J, Moller AB, Zhang J, Gülmezoglu AM, Torloni MR. The Increasing Trend in Caesarean Section Rates: Global, Regional and National Estimates: 1990-2014. BJOG. 2016;11(2): e0148343. doi.org/10.1371/journal.pone.0148343.

2. Brasil. Governo quer reduzir cesarianas desnecessárias. Brasília; 2016 [acesso em 5 dez 2017]. Disponível em: http://www.brasil. gov.br/saude/2016/04/governo-federal-quer-reduzir-cesarianadesnecessaria.

3. Brasil. Diretrizes nacionais de assistência ao parto normal. Brasília: Ministério da Saúde; 2016 [acesso em 22 dez 2017]. Disponível em: http://conitec.gov.br/images/Consultas/2016/Relatorio_DiretrizPartoNormal_CP.pdf.

4. Riscado LC, Jannotti CB, Barbosa RHS. A decisão pela via de parto no Brasil: temas e tendências na produção da saúde coletiva. Texto \& Contexto Enferm. 2016:25(1):e3570014 [acesso em 5 dez 2019]. Disponível em: http://www.scielo.br/scielo.php?script=sci_ arttext\&pid=S0104-07072016000100501\&lng=pt\&nrm=iso.

5. Gullo CE, Soler, ZASG, Cabrera EMS, Irigoyen BBTJ, Rodrigues DA. Cesárea eletiva no Brasil: imposição da autonomia da mulher ou do poder médico? Enfermagem Brasil. 2020;10(6):362-70 [acesso em 18 ago 2020]. Disponível em: http://www.portalatlanticaeditora.com.br/ index.php/enfermagembrasil/article/view/3887.

6. Brasil. Resolução CNE/CES no 4, de 7 de novembro de 2001. Institui Diretrizes Curriculares Nacionais do Curso de Graduação em Medicina. Diário Oficial da União, Brasília; 2001; Seção 1, p. 38.

7. Brasil. Resolução CNE/CES no 3, de 9 de junho de 2014. Institui Diretrizes Curriculares Nacionais do Curso de Graduação em Medicina e dá outras providências. Diário Oficial da União, Brasília; 23 jun. 2014; Seção 3, p. 3-4.

8. Gilbert ACB, Cardoso MHCA, Wuillaume SM, Jung MP. Discursos médicos em construção: um estudo com residentes em obstetrícia/ ginecologia do instituto Fernandes Figueira/Fiocruz. Rev Bras Educ Med. 2009;33(4):615-23 [acesso em 20 dez 2017]. Disponível em: http://www.scielo.br/scielo.php?script=sci_arttext\&pid=S0100$55022009000400012 \& \operatorname{lng}=$ pt\&nrm=iso.

9. Brasil. Programa de humanização no pré-natal e nascimento. Brasília: Ministério da Saúde; 2000 [acesso em 8 dez 2017]. Disponível em: http://bvsms.saude.gov.br/bvs/publicacoes/parto.pdf.

10. Rios IC. Humanidades e medicina: razão e sensibilidade na formação médica. Ciênc Saúde Colet. 2010;15(1):1725-32 [acesso em 8 dez 2017]. Disponível em: http://www.teses.usp.br/teses/disponiveis/5/5137/tde27082010-143815/en.php.

11. Almeida IMQ, Silva FA. Ingressantes no curso de Medicina de uma instituição de ensino superior pública. Ver Ciênc Estud Acad Med. 2017;1(8):10-9 [acesso em 28 out 2019]. Disponível em: https://periodicos. unemat.br/index.php/revistamedicina/article/view/2162/2093.

12. Vaz ALDL, Gléria VO, Bastos GCFC, Sousa IF, Silva AMTC, Almeida RJ. Fatores associados aos níveis de fadiga e sonolência excessiva diurna em estudantes do internato de um curso de Medicina. Rev Bras Educ Med. 2020;44(1):e011 [acesso em 21 maio 2020]. Disponível em: https://www.scielo.br/pdf/rbem/v44n1/19815271-rbem-44-01-e011.pdf.

13. Rios IC. Humanidades e medicina: razão e sensibilidade na formação médica. Ciênc Saúde Colet. 2010;15(1):1725-32 [acesso em 16 maio 2020]. Disponível em: http://www.scielo.br/pdf/csc/v15s1/084.pdf.

14. Ayres JRC, Rios IC, Schraiber LB, Falcão MT, Mota A. Humanidades como disciplina da graduação em Medicina. Rev Bras Educ Med. 2013;37(3):455-63 [acesso em 9 out 2019]. Disponível em: http://www.scielo.br/scielo.php?script=sci arttext\&pid=S010055022013000300019\&lng=en\&nrm=iso.

15. Silva CLA, Cavalcante GF, Ramos LG, Anfe MLS, Castro MR, Mello TRC, et al. Humanização no ensino de graduação no curso de medicina. Diálogos Interdisciplinares. 2019;8(10):118-32 [acesso em 21 maio 2020]. Disponível em: https://revistas.brazcubas.br/index. $\mathrm{php/dialogos/article/view/836.}$

16. Brasil. Lei $n^{\circ} 11.108$, de 7 de abril de 2005. Altera a Lei $n^{\circ} 8.080$, introduzindo o direito ao acompanhante de escolha durante o trabalho de parto, no parto e no pós-parto imediato. Diário Oficial da União; 7 abr 2005.

17. Gomes IEM, Padoin SMM, Langendorf TF, Paula CC, Gomes CA, Ribeiro AC. Benefícios da presença do acompanhante no processo de parto e nascimento: revisão integrativa. Rev Enferm UFSM. 2019;9:61-79 [acesso em 21 maio 2020]. Disponível em: https:// periodicos.ufsm.br/reufsm/article/view/34170/pdf.

18. World Health Organization. WHO recommendations: intrapartum care for a positive childbirth experience. Geneva: WHO; 2018 [acesso em 19 maio 2020]. Disponível em: https://www.who.int/ publications-detail/9789241550215.

19. National Collaborating Centre for Women's and Children's Health. Caesarean section. London: RCOG Press; 2011 [acesso em 19 maio 2020]. Disponível em: https://pubmed.ncbi.nlm.nih. gov/23285498/.

20. Kottwitz F, Gouveia HG, Gonçalves AC. Via de parto preferida por puérperas e suas motivações. Esc Anna Nery Rev Enferm. 2018;22(1):1-8 [acesso em 20 maio 2020]. Disponível em: http://www.scielo.br/scielo.php?script=sci_arttext\&pid=S1414$81452018000100201 \& \operatorname{lng}=$ en\&nrm=iso.

21. Brasil. Diretriz Nacional de Assistência ao Parto Normal. Brasília: Ministério da Saúde; 2016 [acesso em 22 dez 2017]. Disponível em: http://conitec.gov.br/images/Consultas/2016/Relatorio_DiretrizPartoNormal_CP.pdf.

22. Amorim MMR, Souza ASR, Porto AMF. Indicações de cesariana utilizadas em uso: parte I. Femina. 2010;38(8):417-22 [acesso em 16 maio 2020]. Disponível em: http://bhpelopartonormal.pbh.gov.br/estudos_ cientificos/arquivos/cesariana_baseada_evidencias_parte_I.pdf.

23. Noronha Neto C, Souza ASR, Amorim MMR. Tratamento da pré-eclâmpsia baseado em evidências. Rev Bras Ginecol Obstet. 2010;32(9):459-68 [acesso em 26 out 2019]. Disponível em: http://conitec.gov.br/images/Consultas/2016/Relatorio_DiretrizPartoNormal_CP.pdf.

24. Miranda FFS, Toth MVB, Costa TR, Freitas RF. Pré-eclâmpsia e mortalidade materna. Cadernos de Medicina - UNIFESO. 2019;2(1):63-73 [acesso em 10 set 2020]. Disponível em: http:// www.revista.unifeso.edu.br/index.php/cadernosdemedicinaunifeso/ article/view/1296.

25. Alexopoulos, AS, Blair R, Peters AL. Management of preexisting diabetes in pregnancy: a review. JAMA. 2019;321(18):1811-9 [acesso

$11 \mid$\begin{tabular}{l|l} 
REVISTA BRASILEIRA DE EDUCAÇÃO MÉDICA \\
$44(4):$ el67; 2020
\end{tabular} 
em 9 out 2019]. Disponível em: https://jamanetwork.com/journals/ jama/article-abstract/2733213.

26. Souza FMLC, Santos WN, Santos RSC, Rodrigues OB, Santiago JCD, Silva RAR. Tecnologias apropriadas ao processo do trabalho de parto humanizado. Enferm Foco. 2019;10(2):118-124 [acesso em 19 maio 2020]. Disponível em: http://revista.cofen.gov.br/index.php/ enfermagem/article/view/2180.

27. Lansky S, Souza KV, Peixoto ERM, Oliveira BJ, Diniz CSG, Vieira NF, et al. Violência obstétrica: influência da Exposição Sentidos do Nascer na vivência das gestantes. Ciênc Saúde Colet. 2019; 24(8):2811-24 [acesso em 19 out 2019]. Disponível em: http://www.scielo.br/scielo.php?script=sci_ arttext\&pid=S1413-81232019000802811\&lng=en\&nrm=iso.

28. Zanardo GLP, Uribe MC, Nadal AHR, Habigzang LF. Violência obstétrica no brasil: uma revisão narrativa. Psicol Soc. 2017;29e155043 [acesso em 20 out 2019]. Disponível em: http://www.scielo.br/scielo.php?script=sci_ arttext\&pid=S0102-71822017000100218\&lng=en\&nrm=iso.

29. Kopereck CS, Matos GC, Soares MC, Escobal APL, Quadro PP, Cecagno $S$. A violência obstétrica no contexto multinacional. Rev Enferm UFPE. 2018;12(7):2050-60 [acesso em 19 out 2019]. Disponível em: https:// periodicos.ufpe.br/revistas/revistaenfermagem/article/view/231399.

30. Paulino RFOS, Barros SS, Falcão, TT. Violência obstétrica: uma revisão bibliográfica sobre a assistência humanizada no parto. Saber Científico. 2019;8(2):124-34 [acesso em 20 out 2019]. Disponível em: http://revista.saolucas.edu.br/index.php/resc/article/view/1176/pdf.

31. Rodrigues DP, Alves VH, Vieira RS, Leão DCMR, Paula E, Pimentel MM. A violência obstétrica no contexto do parto e nascimento. Rev Enferm UFPE. 2018;12(1):236-46 [acesso em 31 out 2019]. Disponível em: https://periodicos.ufpe.br/revistas/revistaenfermagem/article/ viewFile/23523/26086.

32. Oliveira MC, Merces MC. Perceptions on obstetric violence in the puerperas view. Journal of Nursing UFPE. 2017;11(6):2483-9 [acesso em 19 out 2019]. Disponível em: https://periodicos.ufpe.br/revistas/ revistaenfermagem/article/view/23415/19090.

33. Segundo AFPS, Neta DTC. Clampeamento tardio do cordão umbilical e os benefícios ao neonato: revisão integrativa da literatura. Journal of Specialist. 2019;1(3):1-10 [acesso em 25 out 2019]. Disponível em: http://journalofspecialist.com/jos/index.php/jos/article/view/103.

34. Brasil. Além da sobrevivência: práticas integradas de atenção ao parto, benéficas para a nutrição e a saúde de mães e crianças. Brasília: Ministério da Saúde; 2011 [acesso em 25 out 2019]. Disponível em: https://www. sbp.com.br/fileadmin/user_upload/pdfs/Alem_sobrevivencia_Praticas_ integradas_atencao_parto.pdf.

35. Brasil. Diretrizes de atenção à gestante: a operação cesariana. Brasília: Ministério da Saúde; 2016 [acesso em 19 maio 2020]. Disponível em: http://conitec.gov.br/images/Relatorios/2016/Relatorio_DiretrizesCesariana_final.pdf.
36. Russo JA, Nucci MF. Parindo no paraíso: parto humanizado, ocitocina e a produção corporal de uma nova maternidade. Interface (Botucatu). 2020;24e180390 [acesso em 19 maio 2020]. Disponível em: https://www. scielo.br/scielo.php?pid=S1414-32832020000100213\&script=sci_arttext.

37. Moura TR, Nunes EFC, Latorre GFS, Vargas MM. Dispareunia relacionada à via de parto: uma revisão integrativa. Rev Ciênc Méd. 2018;27(3):157-65 [acesso em 21 maio 2020]. Disponível em: https:// seer.sis.puc-campinas.edu.br/seer/index.php/cienciasmedicas/ article/view/4283.

38. Guiraldello L, Lascala MR, Green MCTP. Análise da frequência e percepção sobre violência obstétricas e suas repercussões ético-legais. Nucleus. 2019;16(1):299-315 [acesso em $1^{\circ}$ nov 2019]. Disponível em: http://www.nucleus.feituverava.com.br/index.php/nucleus/article/ view/3534.

39. Ferrari J, Lima NM. Atitudes dos profissionais de obstetrícia em relação à escolha da via de parto em Porto Velho, Rondônia, Brasil. Rev Bioét. 2010;18(3):645-59 [acesso em $1^{\circ}$ nov 2019]. Disponível em: http://revistabioetica.cfm.org.br/index.php/revista_bioetica/article/ view/591/597.

40. World Health Organization. WHO recommendations: non-clinical interventions to reduce unnecessary caesarean sections. Geneva: WHO; 2018 [acesso em 19 maio 2020]. Disponível em: https://www. who.int/reproductivehealth/publications/non-clinical-interventionsto-reduce-cs/en/.

41. Ryding EL, Lukasse M, Kristjansdottir H, Steingrimsdottir T, Schei B. Pregnant women's preference for cesarean section and subsequent mode of birth: a six-country cohort study. J Psychosom Obstet Gynaecol. 2016;371(3):75-83 [acesso em 20 maio 2020]. Disponível em: https://europepmc.org/article/med/27269591.

\section{CONTRIBUIÇÃO DOS AUTORES}

Yasmin Sendrete de Carvalho Oliveira Leite, Erika Cristina Napolitano Giuliano, Sérgio Alves Dias Júnior, Marcela Souza da Silva, Fábio de Souza Terra e Patrícia Mônica Ribeiro foram responsáveis pela conceituação do trabalho, curadoria de dados, análise formal, investigação, metodologia, análise estatística e redação (rascunho original, revisão e edição).

\section{CONFLITO DE INTERESSES}

Os autores declaram não haver conflito de interesses neste estudo.

\section{ENDEREÇO PARA CORRESPONDÊNCIA}

Yasmin Sendrete de Carvalho Oliveira Leite. Avenida Governador Valadares, 462, ap. 202, Centro, Alfenas, MG, Brasil. CEP: 37130-199.

E-mail: yasmin.sendrete@gmail.com 